\title{
CORRELATION BETWEEN STRESS LEVELS AND THE FIBROMYALGIA IMPACT
}

Elisa Noskoski ${ }^{1, \star}$, Christine Maria Kaiser Leitner1, Maikelli Simes ${ }^{1}$, Kenia Rodrigues de Andrade ${ }^{1}$, Samuel Spiegelberg Zuge ${ }^{1}$

1.Universidade Comunitária da Região de Chapecó, Chapecó (SC), Brazil.

*Corresponding author: elisa.noskoski@unochapeco.edu.br

\section{BACKGROUND}

Fibromyalgia is a syndrome characterized by chronic pain, functional disability and psychosomatic manifestations. Such symptoms generate a direct impact on the life and health conditions of fibromyalgia patients. Therefore, it is clear that the impact caused by fibromyalgia can interfere with psychosocial aspects and decrease life quality. Thus, this work aims to relate the fibromyalgia impact with stress and the life quality of these patients.

\section{METHODS}

This is a cross-sectional study with quantitative design. The study population includes participants aged over 18 years, diagnosed with fibromyalgia. Data collection occurred remotely, from a questionnaire formulated in Google Forms, containing: the fibromyalgia impact questionnaire (FIQ), perceived stress scale (PSS-10) and life quality scale (WHOQOL-BREF). Data were analyzed using the software SPSS, verifying the scales mean and standard deviation, likewise the mean comparison using Kruskal-Wallis and Spearman correlation.

\section{RESULTS}

A total of 427 patients with fibromyalgia participated in the research, of which $99.1 \%$ were female, with a mean age of 41.40 . Regarding the scales of evaluation, it was observed that the average stress levels was $27.52( \pm 5.61)$, ranging from 0 to 40 points. As for the assessment of the fibromyalgia impact, an average of $7.75( \pm 0.95)$ was identified, ranging from 0 to 10 . Regarding life quality, the overall mean was $9.45( \pm 3.30)$, and the most affected domain was the physical, with a mean of $8.69( \pm 2.34)$. When relating the impact and stress caused by fibromyalgia, 145 (33.96\%) patients were classified as low impact, 7 (4.83\%) with low stress level, 84 (57.93\%) with moderate and 54 (37.24\%) with high. Furthermore, 282 (66.04\%) patients have high impact, 1 (0.35\%) with low, 73 (25.89\%) with moderate and 208 (73.76\%) with high stress levels. When comparing the average stress levels and life quality with the fibromyalgia impact (stratified into high and low impact), it is clear that people with a high impact of the disease had higher stress levels and lower quality life levels. Regarding correlations, it was possible to identify a significant correlation between the fibromyalgia impact with stress and life quality. The greater the fibromyalgia impact, the higher the stress levels, and the lower the life quality levels.

\section{CONCLUSION}

It is concluded that the impact of fibromyalgia is related to stress levels and life quality of patients with fibromyalgia. Thus, establishing strategies to minimize the impact of fibromyalgia will allow to reduce stress levels and improve life quality.

\section{KEYWORDS}

Life quality, Impact, Pain. 